\title{
Roughening of cobalt thin films on sapphire (110) upon annealing and superparamagnetic behavior of cobalt nanodots on sapphire (001)
}

Jorge Espinosa

West Virginia University

Follow this and additional works at: https://researchrepository.wvu.edu/etd

\section{Recommended Citation}

Espinosa, Jorge, "Roughening of cobalt thin films on sapphire (110) upon annealing and superparamagnetic behavior of cobalt nanodots on sapphire (001)" (2005). Graduate Theses, Dissertations, and Problem Reports. 2178.

https://researchrepository.wvu.edu/etd/2178

This Thesis is protected by copyright and/or related rights. It has been brought to you by the The Research Repository @ WVU with permission from the rights-holder(s). You are free to use this Thesis in any way that is permitted by the copyright and related rights legislation that applies to your use. For other uses you must obtain permission from the rights-holder(s) directly, unless additional rights are indicated by a Creative Commons license in the record and/ or on the work itself. This Thesis has been accepted for inclusion in WVU Graduate Theses, Dissertations, and Problem Reports collection by an authorized administrator of The Research Repository @ WVU. For more information, please contact researchrepository@mail.wvu.edu. 


\title{
Roughening of cobalt thin films on sapphire (110) upon annealing and superparamagnetic behavior of cobalt nanodots on sapphire (001)
}

\author{
By \\ Jorge Espinosa \\ Thesis Submitted to the Eberly College of Art and Sciences \\ at \\ West Virginia University \\ in partial fulfillment of the requirements for the degree of
}

\author{
Master of Science in Physics \\ David Lederman, Ph D. Chair \\ Mohindar Seehra, Ph.D. \\ Thomas H. Myers, Ph.D. \\ Charter Stinespring, Ph.D. \\ Department of Physics \\ Morgantown, West Virginia University
}

2004

Keywords

Superparamagnetism, nanoparticles, sapphire, cobalt, magnetism, SQUID magnetometry, atomic force microscopy, self-assembly, morphology, surface roughening 


\section{Abstract \\ Roughening of cobalt thin films on sapphire (110) upon annealing and superparamagnetic behavior of cobalt nanodots on sapphire (001)}

\section{Jorge Espinosa}

Atomic force microscopy (AFM) was employed to investigate the surface roughness of $5.0 \mathrm{~nm}$ thick Co films as a function of the annealing temperature. Films were epitaxially grown on (110) sapphire via molecular beam epitaxy (MBE) and magnetron sputtering. Images were acquired in-situ at temperatures ranging between room temperature and $600{ }^{\circ} \mathrm{C}$. Surface roughening starts at $425{ }^{\circ} \mathrm{C}$ and increases depending on the final annealing temperature and time. The films did not show a reversal of the roughening process upon sample cooling. Also a sub-monolayer film of $\mathrm{Co}$ was grown on $\mathrm{Al}_{2} \mathrm{O}_{3}(0001)$ via $\mathrm{MBE}$ at room temperature. The film annealed at $400{ }^{\circ} \mathrm{C}$ revealed the formation of dots with an average diameter of $17.6 \mathrm{~nm}$ and a height of $0.3 \mathrm{~nm}$. Dots showed a superparamagnetic behavior with a blocking temperature of $130 \mathrm{~K}$ and an enhancement of the atomic magnetic moment over the bulk of approximately $9 \%$. 


\section{Acknowledgments}

I thanks my advisor for guiding me and sometimes "pushing" me to accomplish this goal, I thanks my family that supported me in the distance, to my "long term" friend (almost brother) Erie, Shi, Yikuan, Vivek and all my classmates in the department, to the people of the mechanic shop (for helping me on do not cut my fingers), to Dr. Stinespring and Charlie that helped me with some process of my samples and to Monia for all her love and good food all this years. 


\section{Contents}

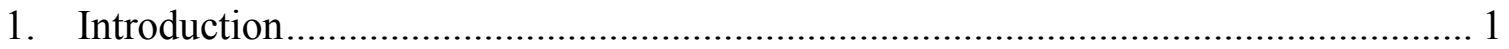

1.1. Techniques for creating magnetic nanostructures.............................................. 2

1.2. Magnetic properties of cobalt ultra-thin films and nano-structures.......................... 5

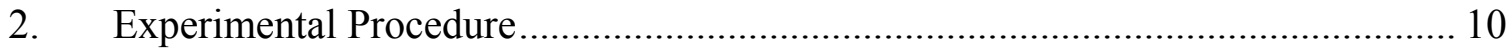

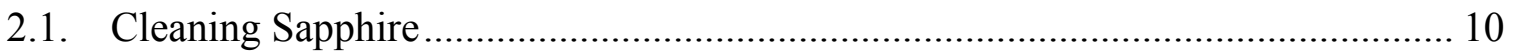

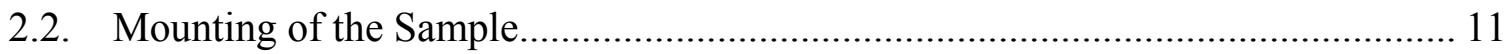

2.3. Cobalt Thin Film Growth............................................................................. 11

2.4. Non Contact Atomic Force Microscopy NC-AFM …………………………….... 13

2.5. Temperature Calibration ................................................................................ 14

2.6. Annealing of Samples ................................................................................ 15

2.7. Magnetic Measurements with the Superconducting Quantum Interference Device

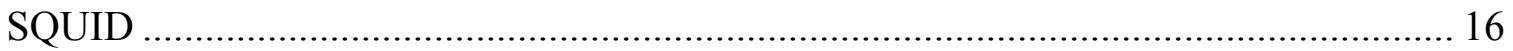

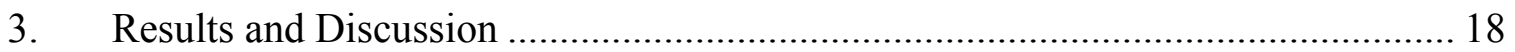

3.1. Roughening of Cobalt Thin Films ................................................................. 18

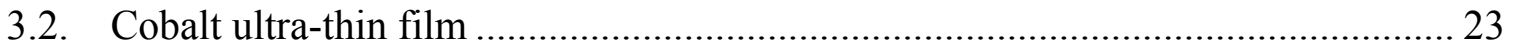

3.2.1. Magnetic Properties of Ultra-Thin film ............................................................ 25

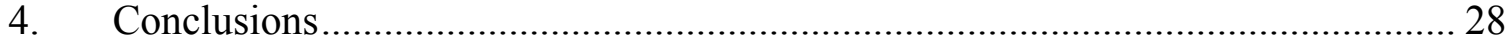

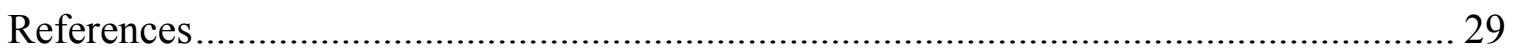

\section{Figures}

Figure 1 AFM image at room temperature of Cobalt over $\mathrm{Al}_{2} \mathrm{O}_{3}(110)$ annealed at 550

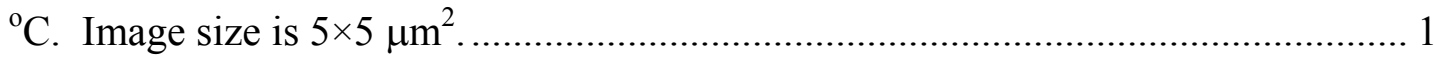

Figure 2 Fe nanowires on top of W(110). Image from Ref. 14...................................... 3

Figure 3 Corundum structure. The c-axis unit cell is $1.3 \mathrm{~nm}$......................................... 4

Figure 4 AFM image of the surface of sapphire (0001) after annealing in air at $1400^{\circ} \mathrm{C}$ for 1 hour. The bottom graph indicates the profile along the blue arrow on the image. ... 4 Figure 5 Step-flow growth. Ideally the atoms would move to the step edges (right picture) after annealing the sample. ............................................................................. 5 
Figure 6. Single domain ellipsoidal particle magnetized at an angle $\alpha$ with respect to the easy axis. 7

Figure 7 Configuration of the chambers for the experiments. 12

Figure 8 Scanning electron microscope image of the needle sensor tip. The apex radius of the tip is $<30 \mathrm{~nm}$. 13

Figure 9 Resonance frequency of the needle sensor as a function of the annealing temperature of the sample for 3 different experiments. 14

Figure 10 Josephson junction. An insulator is placed in between of two superconductor materials.

Figure 11 MPMS SQUID detector diagram. The pick up coil is inductively coupled with the SQUID detector through the isolation transformer and the signal coil (from Quantum Design MPMS Hardware manual).

Figure 12 Wet etched sapphire (11 $\overline{2} 0)$ before deposition of cobalt. Image size is $1 \mu \mathrm{m} \times$ $1 \mu \mathrm{m}$

Figure 13 In-situ AFM images of cobalt (5.0 nm) grown on $\mathrm{Al}_{2} \mathrm{O}_{3}(110)$ at different annealing temperatures. The color scale depicts the height of the islands in an axis perpendicular to the surface of the sample. The size of the images is $1 \mu \mathrm{m} \times 1 \mu \mathrm{m} .20$

Figure 14 Roughening of a cobalt film $(5.0 \mathrm{~nm})$ on sapphire (110). The circles represent the roughness during heating and the dots during cooling.... 22

Figure 15 Roughness as a function of annealing rate for 3 different $5.0 \mathrm{~nm}$ thick Co film samples with different heating rates used to reach the annealing temperature $T_{A} \ldots .22$

Figure 16 AFM image of $\mathrm{Al}_{2} \mathrm{O}_{3}(0001)$ after annealing at $1400{ }^{\circ} \mathrm{C} \times 1$ hour in air. The image size is $2 \mu \mathrm{m} \times 2 \mu \mathrm{m}$.

Figure 17 AFM image of sapphire after growing $0.2 \mathrm{~nm}$ of cobalt at room temperature.

Image size $2 \mu \mathrm{m} \times 2 \mu \mathrm{m}$. 23

Figure 18 AFM image of $0.2 \mathrm{~nm}$ of Cobalt over sapphire (001) after annealing in ultra high vacuum at $400{ }^{\circ} \mathrm{C}$. The image shows the formation of nanodots with an average diameter of $17.7 \mathrm{~nm}$. The image size is $2 \mu \mathrm{m} \times 2 \mu \mathrm{m}$. 24

Figure 19 Histogram of the diameter of the nanodots obtained from the image in Figure 20. Statistics are based on measurement of 50 nanodots. 
Figure 20 AFM image of cobalt nanodots. The image corresponds to the squared region of Figure 18. Image size: $500 \mathrm{~nm} \times 500 \mathrm{~nm}$. Image is slightly filtered.................. 24 Figure 21. Magnetization versus temperature for the cobalt ultra-thin film. The black dots represent the magnetization for the field cooled sample and in white dots the magnetization for zero field cooled. The measurement field was 1000 Oe. 25

Figure 22 Magnetization as a function of magnetic field for the ultra-thin film taken at $300 \mathrm{~K}$. The red line corresponds to the fitting of the Langevin function to the experimental data. 


\section{Introduction}

The creation and manipulation of magnetic structures with dimensions on the order of nanometers is very important from the technological point of view. The information storage media industry needs such structures in order to create new devices capable of recording a higher density of information in order to stay competitive. From a fundamental point of view, magnetic nanostructures are extremely interesting because quantum effects are enhanced and, furthermore, new magnetic structures can form because of the large importance of surface effects. Recently it has been possible to artificially reduce the size of magnetic films (two dimensions) down to one and zero dimensions (in nanowires and nanodots, respectively), hence providing the opportunity of observing new physical properties and quantum effects that cannot be noticed in bulk materials. Usually small magnetic particles behave like single domains particles, leading to a superparamagnetic behavior. ${ }^{1}$ In the superparamagnetic state, the particles behave ferromagnetically below some transition temperature, known as the blocking temperature, and paramagnetically above it. Another interesting phenomena in magnetic ultra-thin films is the enhancement of the magnetic moment per atom. This behavior results from an increase in the spin and orbital moments of the atoms located at surfaces. ${ }^{2}$

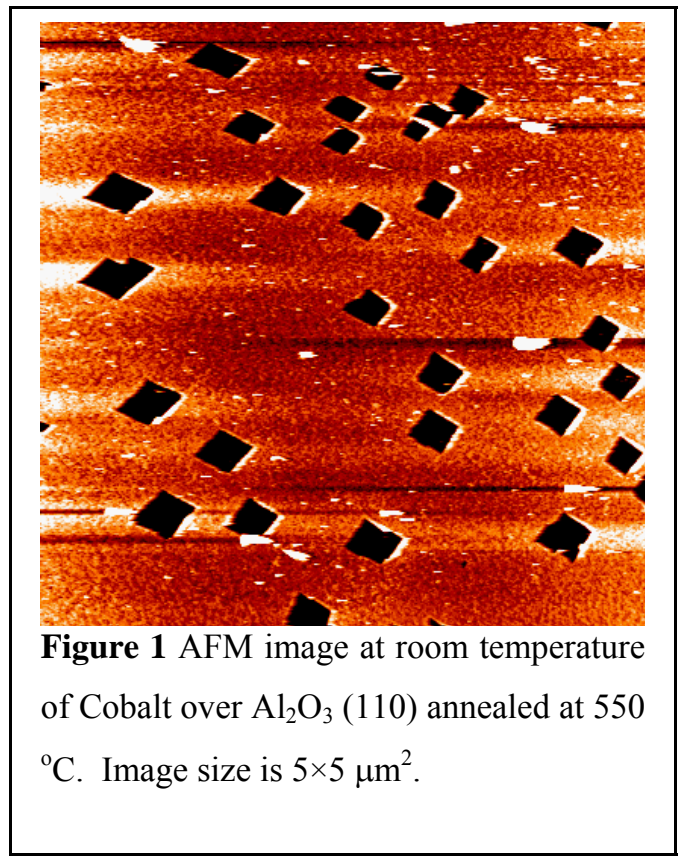

Cobalt is a ferromagnetic transition metal with a curie temperature of $1388 \mathrm{~K}$ and it undergoes a martensitic transition from the hcp to fcc structure at a temperature of 415 ${ }^{\circ} \mathrm{C} .{ }^{3}$ Cobalt thin films have been extensively studied in different substrates. In sapphire (110) it is well known that crystallinity and surface smoothness of cobalt films grown using sputtering reach their maximum at 300 ${ }^{\circ} \mathrm{C} .{ }^{4}$ In recent years cobalt films deposited on sapphire have been examined using atomic 
force microscopy (AFM). In one particular experiment performed by our group, it was found that thin Co films (thickness $t<20 \mathrm{~nm}$ ) grown via sputtering undergo a sudden reentrant smoothening at $500{ }^{\circ} \mathrm{C}$ that coincides with a recrystallization of the lattice from a fcc (111) orientation to a fcc (001) orientation. ${ }^{5,6}$ Also, the films showed the formation of rectangular pits with sides as small as $100 \mathrm{~nm}$ when coinciding with the smoothening transition, as shown in Figure 1. Higher annealing temperatures increase the roughness once again. The physical origins of the pit formation and the smoothening transition are not well understood. For this reason, the study of this process requires controlled experiments where we are able to grow the sample in an ultraclean environment and to observe the surface of our samples with spatial resolution of nanometers at high temperatures.

In the present work, we grew cobalt over sapphire (110) in ultra high vacuum (UHV) using molecular beam epitaxy (MBE) to study the roughness transition. The thickness of the samples ranged from $2 \mathrm{~nm}$ to $8 \mathrm{~nm}$. The morphology of the samples was analyzed using a non-contact atomic force microscope (NC-AFM) with the capability of imaging the surface at temperatures above room temperature. In another experiment, we evaporated a sub-monolayer film of Cobalt on faceted $\mathrm{Al}_{2} \mathrm{O}_{3}$ (0001) using MBE. We studied the magnetic properties of ultra-thin film of cobalt and its suitability to form structures with sizes at the nanometer scale. We demonstrated that magnetic dots spontaneously form upon annealing single-monolayer Co films deposited at room temperature.

\subsection{Techniques for creating magnetic nanostructures}

Different techniques have been employed to produce magnetic nanostructures. Using electron-beam lithography it is possible to obtain patterns in the nanometer scale and using scanning probe microscopy it has been feasible to go even further down to atomic scale features. ${ }^{7}$ Unfortunately these techniques produce patterns in small areas only and the time required to generate them is extremely long. Alternative procedures consist of creating self-organized surfaces and then depositing magnetic materials on top of these surfaces. In this case, the organization of the particles is determined by the structure of 
the template under the right conditions ${ }^{8}$. The first attempts at creating such structures were made using porous alumina templates, created by anodizing aluminum in acidic electrolytes. ${ }^{9}$ This produces cylindrical pores with diameters of $4 \mathrm{~nm}$ to $200 \mathrm{~nm}$. Fe, Ni and Co nanowires have been prepared inside these pores using electrochemical plating, ${ }^{10,11}$ which produces nanostructures with well controlled diameters. Applying the same idea, other groups have used polycarbonate membrane pores to produce cobalt nanowires $^{12}$, but the pore diameter obtained with this method is approximately $200 \mathrm{~nm}$, much larger than the structures produced with porous alumina. Other techniques include using Argon ion sputtering for milling the surface of thin films, for example to fabricate nanowires of $\mathrm{Co}$ deposited on $\mathrm{Cu}(001){ }^{13}$

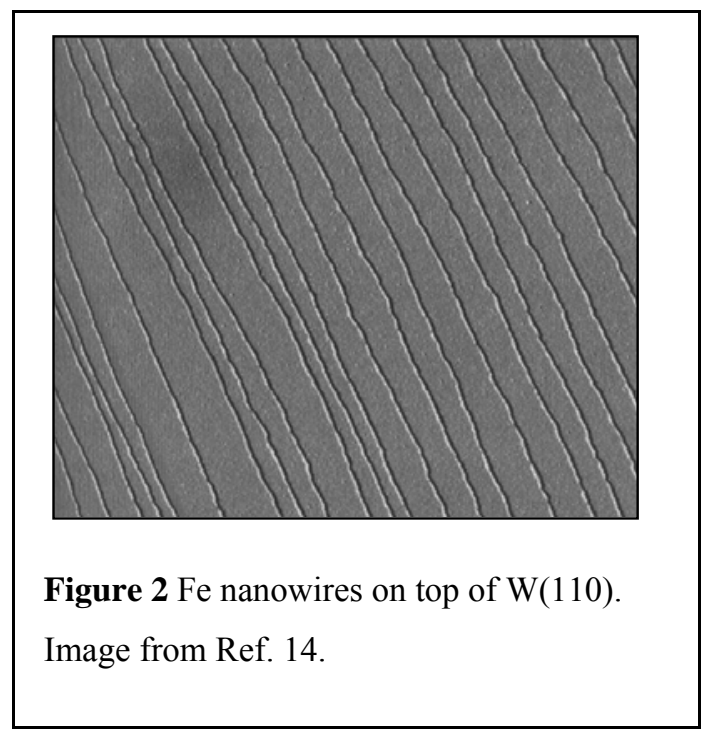

Another approach is to exploit the surface morphology of substrates prepared at high temperature. Fe nanostrips were prepared on stepped W (110) substrates $^{14}$ (see Figure 2), and another group has fabricated Fe nano-stripes on highly faceted $\mathrm{Al}_{2} \mathrm{O}_{3}$ (100) by evaporating the metal at an angle of $5^{\circ}$ with respect to the surface of the substrate. ${ }^{8}$

In this work, we explored the possibility of preparing cobalt nanostructures on faceted $\mathrm{Al}_{2} \mathrm{O}_{3}(001)$. Sapphire is an excellent substrate because, as an oxide, it is not sensitive to contamination, and is highly inert. Sapphire has the corundum crystal structure shown in Figure 4. The unit cell is composed of 18 layers, 6 layers of $\mathrm{O}$ atoms and 12 layers of $\mathrm{Al}$ atoms. The c axis unit cell is $1.30 \mathrm{~nm}$ long. ${ }^{15}$ 


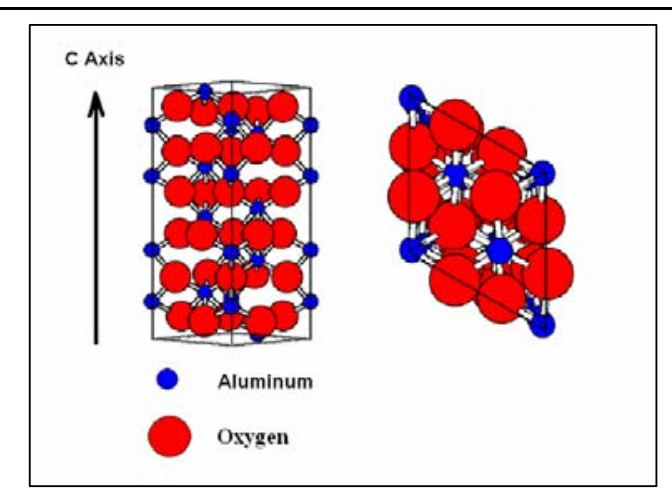

Figure 4 Corundum structure. The c-axis unit cell is $1.3 \mathrm{~nm}$.

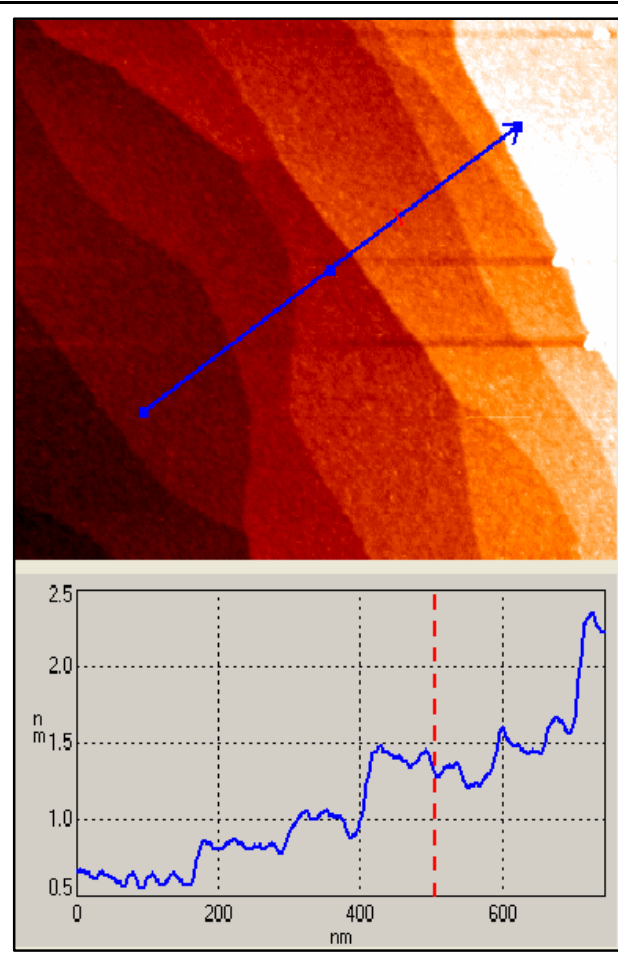

Figure 3 AFM image of the surface of sapphire (0001) after annealing in air at $1400^{\circ} \mathrm{C}$ for 1 hour. The bottom graph indicates the profile along the blue arrow on the image.

5). This method is called step-flow growth and in principle the stripe width can be

\footnotetext{
${ }^{\mathrm{i}}$ The process for obtaining clean and faceted surfaces on sapphire is given in Section 2.
} 
controlled by the coverage of the evaporated material, and the wire spacing determined by the miscut angle of the substrate or the terrace width of the template.

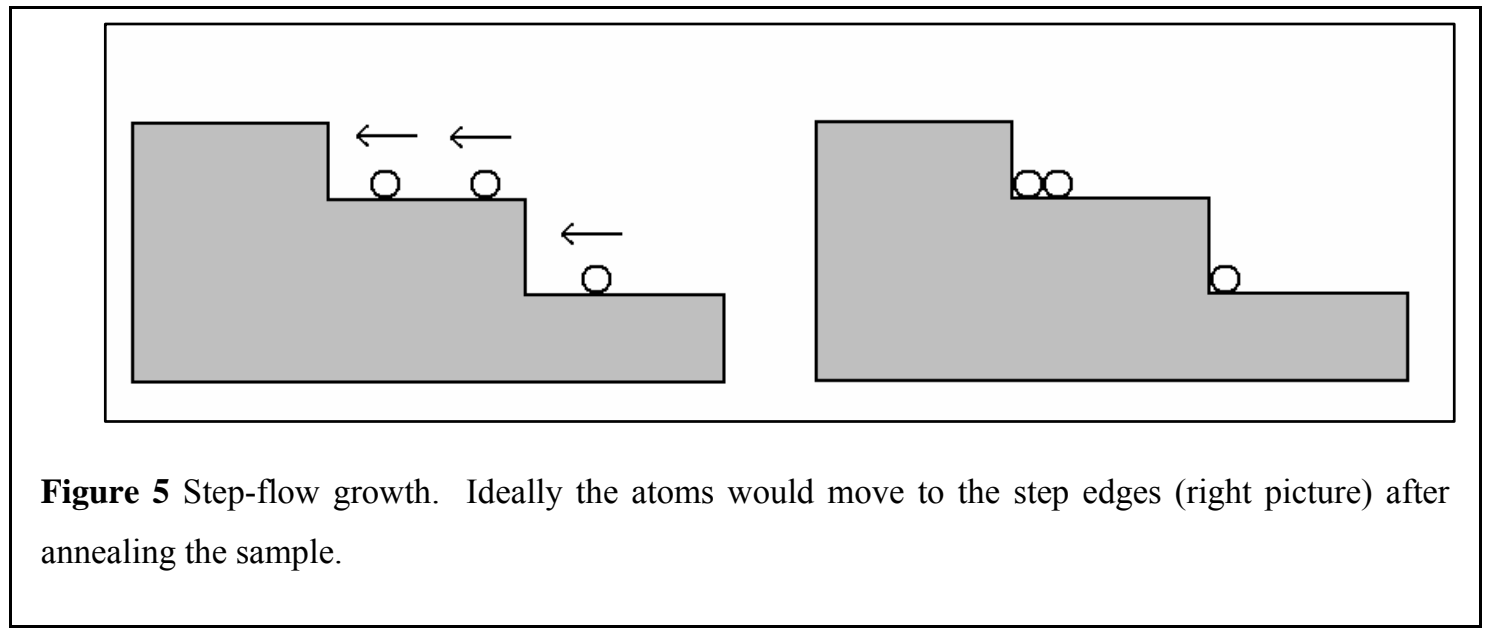

\subsection{Magnetic properties of cobalt ultra-thin films and nano-structures}

Ultra-thin magnetic films (thickness $<1 \mathrm{~nm}$ ) grown on oxide substrates have been investigated in limited cases only. Shiratsuchi et al. ${ }^{20}$ have studied ultra-thin films of Fe on $\mathrm{Al}_{2} \mathrm{O}_{3}(0001)$, reporting a superparamagnetic behavior of the samples.

Since cobalt is a ferromagnetic material, it is useful to present a general description of the ferromagnetism of bulk cobalt and then explain how those properties are changed in ultra-thin films. The free energy of a ferromagnetic material in a magnetic field can be described as the contribution of various terms ${ }^{21}$ :

$$
F_{T}=F_{H}+F_{D}+F_{K}+F_{E}
$$

where $F_{H}$ is the magnetization energy of the material when a magnetic field $\mathbf{H}$ is applied, and can be expressed as:

$$
F_{H}=-\int \mathbf{M} \cdot \mathbf{H} d V
$$


$F_{D}$ is the self-energy of the magnetization in its own magnetostatic field, $\mathbf{H}_{s}$ is the field of the surface that can be write down as:

$$
F_{D}=-\frac{1}{2} \int \mathbf{M} \cdot \mathbf{H}_{\mathbf{s}} d V
$$

where $\boldsymbol{H}_{s}=-D M$ and D is the demagnetization coefficient.

$F_{K}$ is the crystalline anisotropy of the material. For cobalt with hexagonal structure it is known that its easy axis lies along the c-axis. For an angle $\theta$ between the magnetization direction and the easy axis, the anisotropy energy can be described by the equation:

$$
F_{K}=K_{1}^{\prime} \sin ^{2} \theta+K_{2}^{\prime} \sin ^{4} \theta
$$

$K_{1}$ and $K_{2}$ are the anisotropy constants. For cobalt the anisotropy constants are $K_{1}=$ $4.1 \times 10^{6} \mathrm{ergs} / \mathrm{cm}^{3}$ and $K_{2}=1.0 \times 10^{6} \mathrm{ergs} / \mathrm{cm}^{3}$. Finally the term $F_{E}$ is exchange interaction, responsible of the Weiss molecular field that explains the ferromagnetism, which can be expressed as:

$$
F_{E}=-\sum_{i j} J_{i j} \mathbf{S}_{i} \cdot \mathbf{S}_{j}
$$

$J_{i j}$ is the exchange interaction and $\mathbf{S}_{\mathbf{i}}$ and $\mathbf{S}_{\mathrm{j}}$ are localized spins in the lattice. The interaction exchange $J_{i j}>0$ for ferromagnets and $J_{i j}<0$ for antiferromagnets. 
It is well known that the demagnetization energy $F_{D}$ is minimized if the ferromagnetic material is composed of magnetic domains. ${ }^{21}$ In large samples, $F_{D}$ dominates over $F_{E}$ because $F_{E}$ acts primarily over nearest neighbors and $F_{D}$, being a dipole interaction, decays much more slowly. Generally magnetic domains are arranged in a random fashion and the net magnetization of the material might be zero. If an

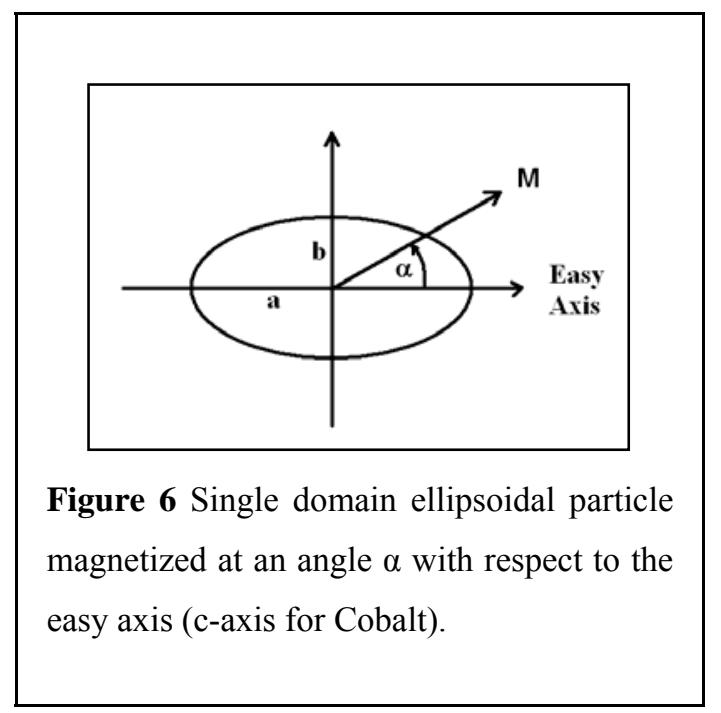

external magnetic field is applied to the ferromagnet, the magnetization of each domain will tend to align in the direction of the field. When all the domains are aligned in the same direction we talk about the saturation of the material. Sometimes we can find materials behaving as single domains, this happens for example when a specimen was magnetized to saturation or when the material is composed of very small particles. Let us consider a cobalt single-domain ellipsoidal particle with the c-axis parallel to the mayor semi-axis as in Figure 6:

We can calculate the energy of demagnetization per volume unit for the single domain ellipsoidal particle using equation (3):

$$
F_{D}=\frac{1}{2}\left(D_{a}[M \cos \alpha]^{2}+D_{b}[M \sin \alpha]^{2}\right)
$$

$D_{a}$ and $D_{b}$ are the demagnetization coefficients along the $a$ and $b$ axis and $M$ is the magnetization for the particle. After some algebra we obtain: 


$$
F_{D}=\frac{1}{2} M^{2} D_{a}+\frac{1}{2} M^{2}\left(D_{b}-D_{a}\right) \sin ^{2} \alpha
$$

Therefore comparing eqs. (4) and (7) we can say that the energy of the particle of volume $\mathrm{V}$ having an angular dependence with $\alpha$ is ${ }^{21}$ :

$$
F_{T}=K_{e f f} V \sin ^{2} \alpha
$$

where $V$ is the volume of the particle and $K_{\text {eff }}=M^{2}\left(D_{b}-D_{a}\right)$ for the demagnetization factor energy or $K_{\text {eff }}=2 K_{l}$ for the crystalline anysotropy. The energy will be a minimum for $\alpha=0$ or 180 degrees. Also the magnetization will remain stable along these directions unless energy is given to the system to overcome the potential barrier equal to $K_{\text {eff }} V$ for $\alpha$ $=\pi / 2$. This might happen if the particle volume decreases or by increasing the temperature of the sample. Under these conditions, the magnetization of the particles might switch spontaneously. The critical temperature for this spontaneous switching of magnetization is called the blocking temperature $\left(T_{B}\right)$. Below $T_{B}$, the particles behave ferromagnetically and above the temperature they behave as paramagnets. If there are interactions between the particles, each particle acts as a single spin (composed of the total moment of all the magnetic atoms that make up each nanoparticle), and the entire system behaves as a paramagnet. Particles that show this kind of behavior are called superparamagnetic.

In a submonolayer thin film of cobalt, the formation of ferromagnetic islands is expected because not all the substrate surface is covered with cobalt atoms. Also in thicker films of cobalt on sapphire, the formation of islands is possible if the film is deposited on the substrate at a temperature higher than $300{ }^{\circ} \mathrm{C} .{ }^{4}$ Those islands might behave as single domain particles if they are smaller than some critical size. This size can be calculated equating the energy of a single domain particle with the energy of a particle with two domains separated by a Bloch wall. The energy of the wall is obtained evaluating the exchange interaction of all pairs of spins in the transition region between the two domains given by the following equation ${ }^{21}$ : 


$$
F_{\text {wall }}=2 \pi S\left(\frac{J_{e x} K_{1}}{a}\right)^{\frac{1}{2}} \times A
$$

where $S$ is the spin of the atoms, $J_{e x}$ is the exchange energy, $K_{l}$ is the anysotropy constant, $a$ is the lattice parameter and $A$ the area separating both domains. The energy of the single domain particle can be calculated as the square of the saturation magnetization times the volume of the particle. For a spherical particle of cobalt with $S=0.86, a=$ $0.3528 \mathrm{~nm}, J_{e x}=1.8 \times 10^{-14} \mathrm{erg}^{34}, K_{1}=4.1 \times 10^{6} \mathrm{erg} / \mathrm{cm}^{3}$ and $M_{s}=1400$ Oe, the critical diameter of the particle is approximately $60 \mathrm{~nm}$.

The size of a particle in a superparamagnetic state can be estimated from experimental data by measuring magnetization vs. magnetic field above $T_{B}$. As discussed above, for T $>T_{B}$ the particles behave as a collective paramagnet. Therefore, the magnetization as a function of magnetic field behaves like that of a paramagnetic material according to ${ }^{21}$

$$
M=M_{0} B_{J}(y)
$$

$M_{0}$ is the saturation of the magnetization for the paramagnetic particle given by:

$$
M_{0}=N g \mu_{B} J
$$

$N$ is the number of paramagnetic atoms, $g$ is the gyromagnetic ratio, $J$ is the total angular momentum and $B(y)$ is the Brillouin function:

$$
\begin{aligned}
& B_{J}(y)=\left\{\frac{2 J+1}{2 J} \operatorname{coth}\left(\frac{2 J+1}{2 J}\right) y-\frac{1}{2 J} \operatorname{coth} \frac{y}{2 J}\right\} \\
& y=\frac{J g \mu_{B} H}{k T}=\frac{\mu_{P} H}{k T}
\end{aligned}
$$


where $\mu_{B}$ is the Bohr magneton, $\mu_{P}$ the average magnetic moment per particle, $H$ the magnetic field applied to the sample, $k$ the Boltzmann constant, $T$ the temperature of the particle and $y$ the competition between the magnetic energy and the thermal energy in the material. If all orientations of the magnetic moments are allowed, this is equivalent to $J \rightarrow$ $\infty$, the Brillouin function becomes the Langevin function $L(y)$ so that:

$$
\underset{J \rightarrow \infty}{B_{J}(y)}=\operatorname{coth} y-\frac{1}{y}=L(y)
$$

By fitting the magnetization curves versus magnetic field to the Langevin function, and using the average magnetic moment per particle $\mu_{P}$ as a parameter, ${ }^{22}$ it is possible to determine the number of Bohr magnetons contained in each nanoparticle.

\section{Experimental Procedure}

\subsection{Cleaning Sapphire}

Obtaining a clean substrate surface is essential for performing controlled growth experiments. There are 2 methods for cleaning sapphire: surface etching using a mixture of acids and an anneal in air above $1100{ }^{\circ} \mathrm{C}$. We used the first method to measure roughening as a function of temperature and the second one for the nanostructure experiments.

The recipe for the wet etching is the following: ${ }^{23}$

1. Degrease the sample using acetone, methanol, and deionized water.

2. Make a solution of sulfuric acid and phosphoric acid 3:1 $\left(\mathrm{H}_{2} \mathrm{SO}_{4}: \mathrm{H}_{3} \mathrm{PO}_{4}\right)$

3. Heat up the mixture up to $160{ }^{\circ} \mathrm{C}$

4. Put the sapphire into the mixture for 10 minutes.

5. Cool down the sapphire and rinse it in deionized water.

The method of annealing only is much easier in principle. The sapphire substrate is first degreased and then placed in an oven for annealing in air. The substrates were 
annealed at $1400{ }^{\circ} \mathrm{C}$ for 1 hour inside an alumina tube and post annealed in UHV at 500 ${ }^{\circ} \mathrm{C}$ using a heating rate of $100{ }^{\circ} \mathrm{C}$ per hour. During this process the pressure on the chamber was kept under $5 \times 10^{-9}$ Torr using a titanium sublimation pump (TSP) in order to avoid contamination of the sapphire surface.

\subsection{Mounting of the Sample}

The substrates were mounted on an Omicron indirect heating plate, which allowed us to simultaneously scan the sample using a non-contact atomic force microscope (NCAFM) while increasing the temperature of the sapphire in UHV. A current passing through a PBN (pyrolytic boron nitride) plate, placed approximately $3 \mathrm{~mm}$ behind the sample, allowed us to heat up the substrate indirectly.

\subsection{Cobalt Thin Film Growth}

The samples were grown in UHV with a base pressure of $2 \times 10^{-9}$ Torr using a molecular beam epitaxy (MBE) system. The e-beam source (MDC e-vap 4000) has a filament that generates a beam of electrons. These electrons are focused on the source material target using a magnetic field. It is necessary to sweep the beam around the material in order to heat up the target homogeneously. We used a circular sweeping pattern for cobalt. The evaporated material is then deposited on our substrate, which is rotating during growth to assure a uniform film. We used different substrate temperatures during growth. For thick cobalt samples we used a temperature of $315^{\circ} \mathrm{C}$ because it is well known that at such growth temperatures the films are smoother. ${ }^{4}$ The thin cobalt samples used on nanostructures were grown at room temperature so as not to introduce any change in the surface before simultaneously annealing and imaging. The growth rate and the thickness of the films were measured using a calibrated quartz crystal monitor. The thickness of our samples ranged between $30 \AA$ to $50 \AA$ for the roughness studies, and was $2 \AA$ (less than one monolayer) for the nanostructure experiments. Once the samples are grown, they are transferred in vacuum to a distribution chamber and then to the AFM chamber, as shown in Figure 7. After the analysis, the samples are transferred 
back to the MBE chamber to cover them with $20 \AA$ of aluminum in order to avoid oxidation when exposed to air.

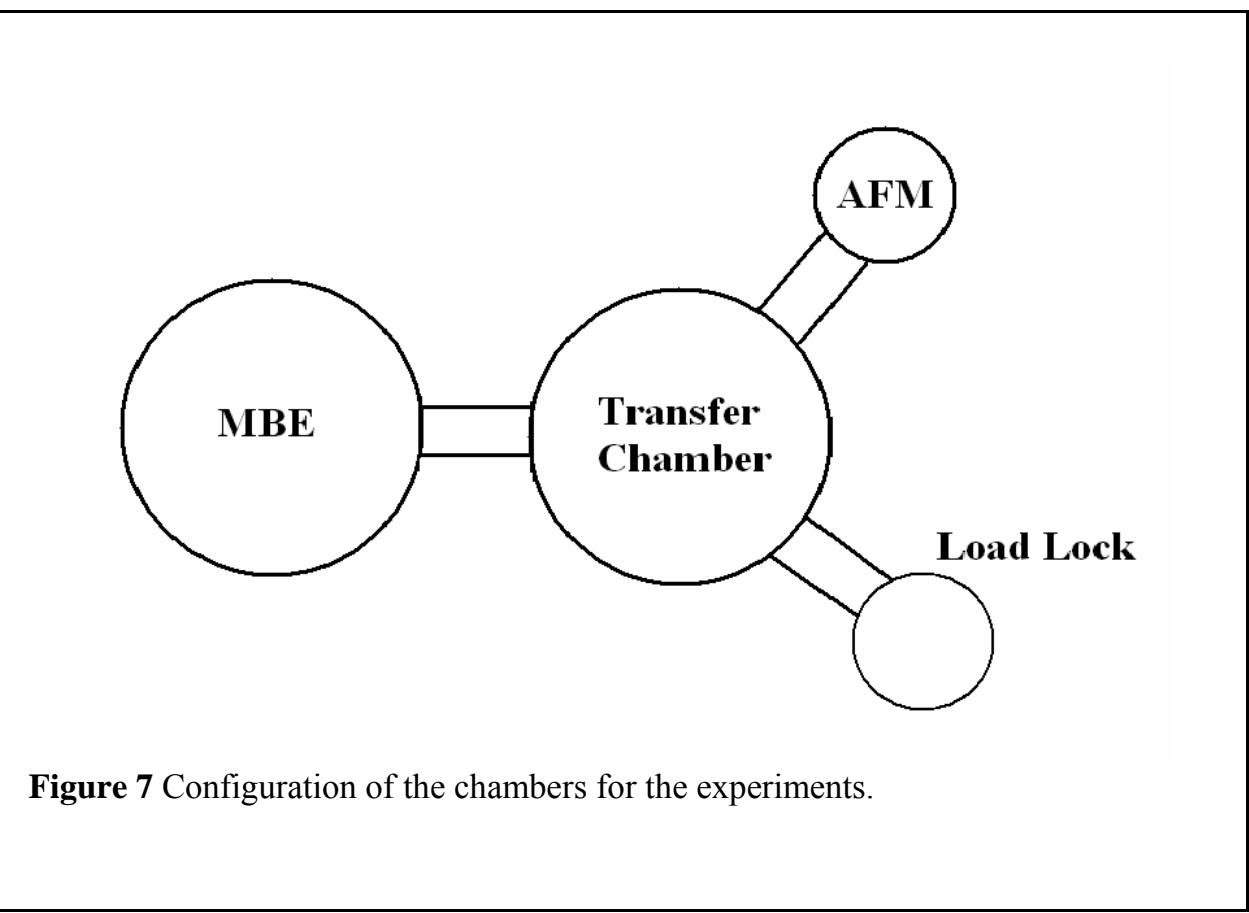




\subsection{Non Contact Atomic Force Microscopy NC-AFM}

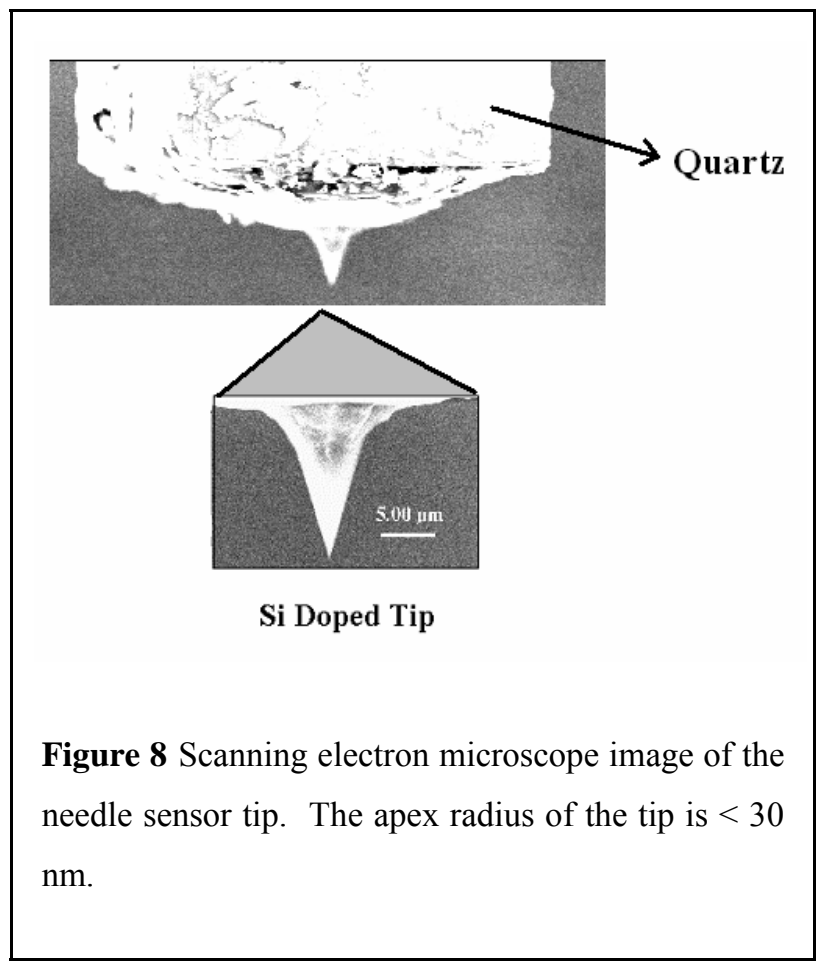

Atomic force microscopy has been used extensively since its development in the 90's, but only recently it has been possible to obtain atomic resolution on $\mathrm{Si}\left(7^{*} 7\right)^{24}$ and insulators like sapphire. ${ }^{25}$ This result is important because in principle we could study insulators with atomic resolution, something that cannot be done with STM. The sensor used in our AFM is called a needle sensor; which consists of a silicon cantilever glued to a quartz resonator with a resonance frequency of approximately

$1 \mathrm{MHz}$, as shown in Figure 8. When this tip is placed near the surface of a sample the sensor undergoes a change in its amplitude of oscillation ${ }^{26}$ and a phase shift. This last parameter gives the highest sensitivity for force microscopy ${ }^{27}$. By scanning the sample tip on the surface of our sample, the tip undergoes different phase shifts depending on how close or how far it is from the sample. Making this phase shift constant, by adjusting the distance from the surface using feedback, causes the tip to move up and down during the scanning process. Recording of the movement of the tip in three dimensions allows the AFM to image the surface of the sample. 


\subsection{Temperature Calibration}

The measurement of the temperature during scanning is not simple because the Omicron microscope does not have a thermocouple near the sample in order to avoid vibrations that could affect the quality of the images. In order to estimate the

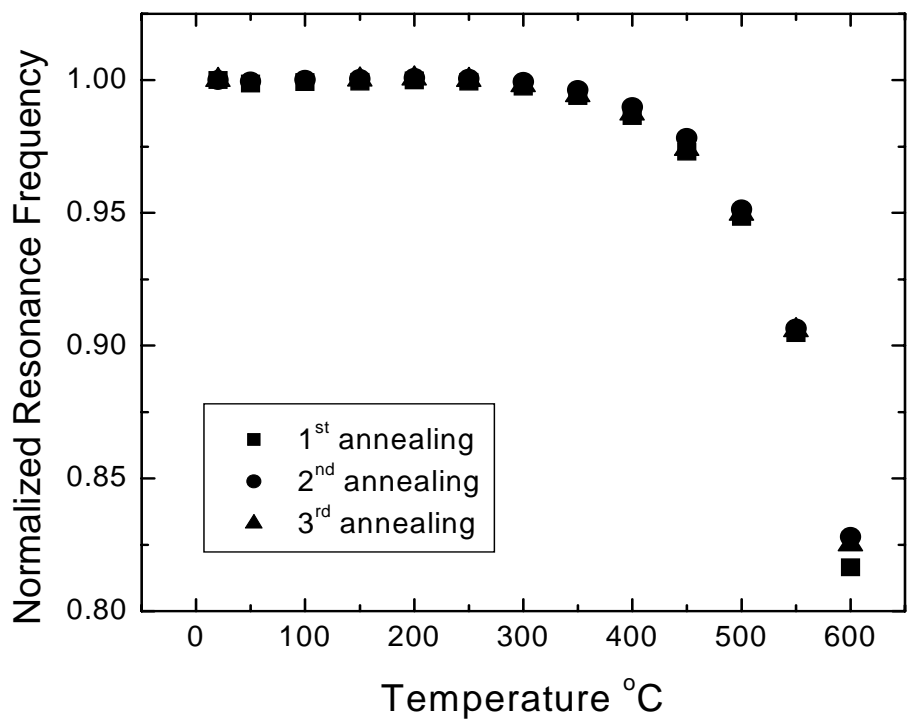

Figure 9 Resonance frequency of the needle sensor as a function of the annealing temperature of the sample for 3 different experiments.

temperature, Omicron included a calibration of the sample temperature as a function of the power applied to the PBN plate. However, during the experiments, we realized that we could use the resonance frequency of the needle sensor as an indicator of the temperature on the sample. Figure 9 shows the resonance frequency of the sensor as a function of temperature for three different experiments using the same needle tip. At temperatures below $300{ }^{\circ} \mathrm{C}$, the frequency is quite constant, but as the temperature increases, the resonance frequency decreases. At $600{ }^{\circ} \mathrm{C}$ the resonance frequency drops drastically and it is impossible to obtain a reliable image above that temperature. This might be caused by a change of stiffness in the needle sensor; above $300{ }^{\circ} \mathrm{C}$ the quartz becomes softer and therefore the resonance frequency decreases. This result is very important because there is no information about the behavior of the needle sensor as a function of the temperature in the literature and also because we can estimate the 
temperature very precisely above $300{ }^{\circ} \mathrm{C}$ where morphology changes start to happen in cobalt films. It also allowed us to determine when the sample had reached thermal equilibrium by monitoring when the frequency stopped changing. It is important to note that although the normalized frequency change is very reproducible, the starting frequency value did vary less than $1 \%$ from run to run. This means that slight irreversible changes in the tip occurred during each heating cycle.

\subsection{Annealing of Samples}

Once the cobalt is grown on the MBE chamber, the sample was transferred to the SPM in order to imaging the surface during annealing. As the images were acquired at high temperature, some care needed to be taken with the needle sensor tip. First, after the temperature was increased, the needle had to be at an imaging distance from the sample at all times, otherwise it would not reach thermal equilibrium and the images were noisy. Also, one had to wait one hour before scanning the surface after increasing the temperature, otherwise a lack of thermal equilibrium could produce a crash of the tip. Finally, the images had to be corrected for thermal drift in the piezos. The AFM can be programmed to automatically shift the tip in the $\mathrm{XY}$ axes in a constant rate. This was carried out entering the $\left(\mathrm{X}_{1}, \mathrm{Y}_{1}\right)$ and $\left(\mathrm{X}_{2}, \mathrm{Y}_{2}\right)$ position for the same pattern in 2 different images; with that information, the program computes the drift it must compensate for by moving the tip in order to track the same feature along different images.

The annealing procedure is the following:

1. Increase the temperature in steps of $50^{\circ} \mathrm{C}$.

2. At each temperature take an image trying to track the same features if possible.

3. Increase the temperature taking images up to $600^{\circ} \mathrm{C}$.

4. Decrease the temperature down to room temperature following the same procedure. 
For the $2 \AA$ cobalt film, we annealed the sample up to $400{ }^{\circ} \mathrm{C}$ (below the temperature at which the roughening transition happens), heating at a rate of $100{ }^{\circ} \mathrm{C}$ per hour.

\subsection{Magnetic Measurements with the Superconducting Quantum Interference Device (SQUID)}

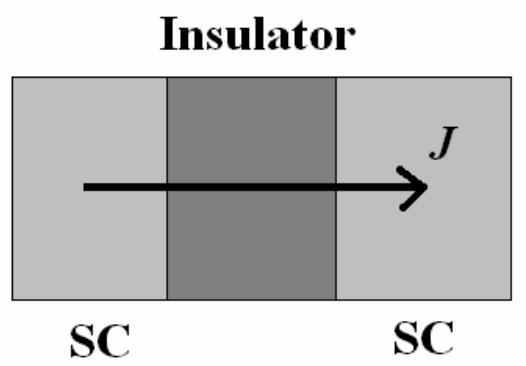

Figure 10 Josephson junction. An insulator is placed in between of two superconductor materials.
The SQUID is the heart of the Quantum Design Magnetic Properties Measurement System (MPMS) that allows us to measure the magnetization of a sample with high accuracy. The system works as follows: a sample is placed at the center of a superconducting coil that produces a magnetic field and induces a magnetization in our sample. The sample already magnetized is moved up

and down inside a superconducting pick up coil. The induced current in the coil is proportional to the magnetization of the sample. The pick up coil configuration is built as a second derivative array, which helps to eliminate uniform drifts from the superconducting magnet and other external magnetic field contributions, such as the Earth's magnetic field. The signal from the pick up coil is then inductively coupled to the SQUID detector through a signal coil attached to a superconducting rf isolation transformer. The SQUID is based on the Josephson effect theoretically proposed in 1962 by Josephson ${ }^{28}$ and experimentally detected in 1963 by Anderson and Rowell. ${ }^{29}$ The Josephson effect can be observed by placing an insulator in between of two superconductors as in Figure 10. In the absence of any external voltage, a current flows across the insulator; this phenomenon is called the DC Josephson effect. If a DC voltage is applied between the junction, then an oscillating current will flow across the insulator given by the equation: ${ }^{30}$ 


$$
J=J_{0} \sin \left(\delta_{0}-\frac{2 e V t}{\hbar}\right),
$$

where $J_{o}$ is the maximum value of the current related with the thickness of the insulators, $\delta_{\mathrm{o}}$ is the phase difference of the currents at both sides of the insulators and $V$ is the voltage across the junction.

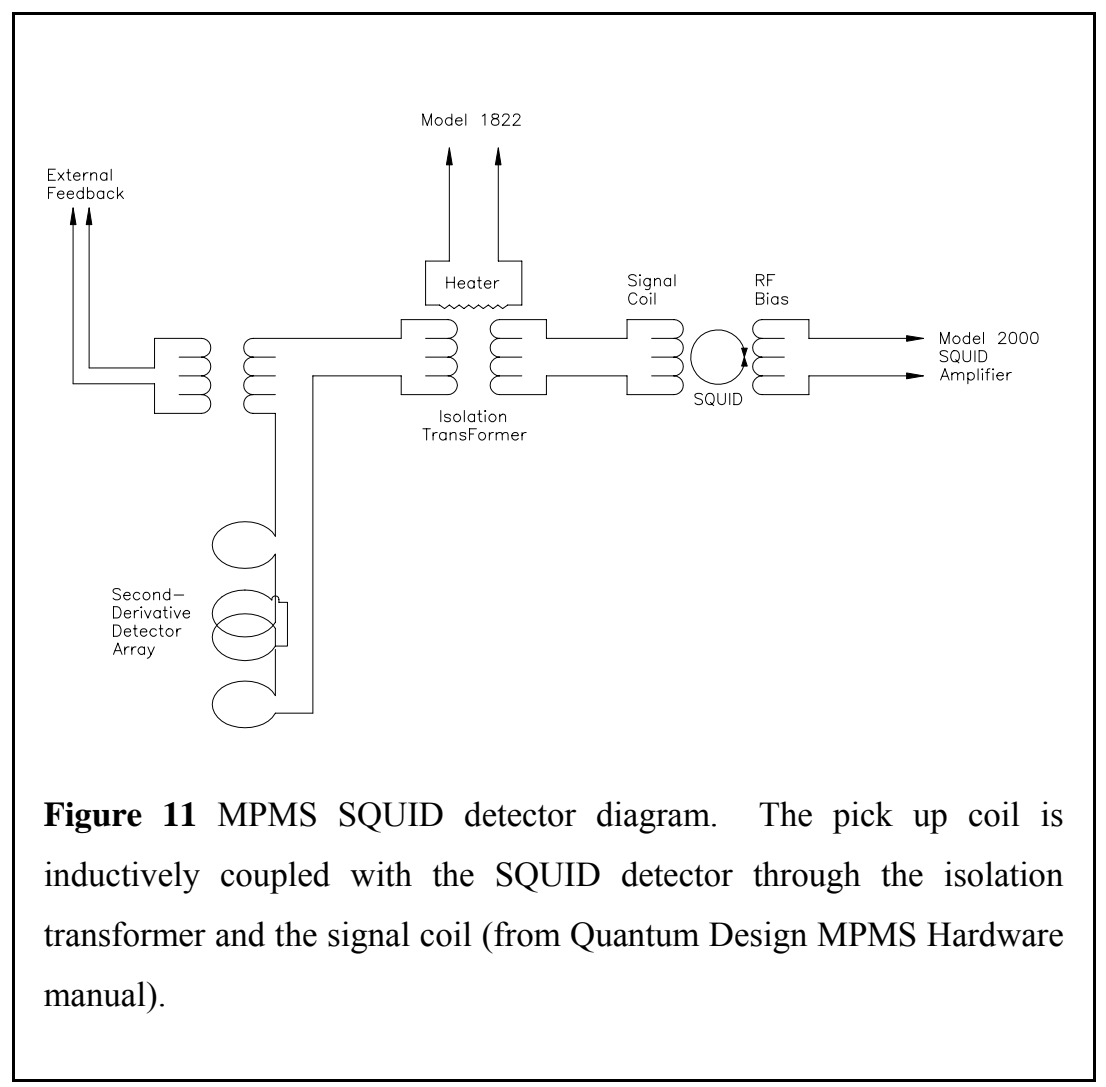

This result is called AC Josephson effect. The MPMS works with the AC Josephson effect, using a single junction into a closed loop supercontuctor coil. ${ }^{31}$ The SQUID is biased with a RF signal in order to overcome the zero voltage region of the Josephson junction as shown in Figure 11. When a magnetic flux crosses the loop, an induced sinusoidal voltage is generated that is periodic in $\Phi_{0}$ the quantum flux $\left(\Phi_{0}=2.03 \times 10^{-15}\right.$ $\mathrm{Wb})$. However, the SQUID can detect a variation in flux $\delta \Phi$ much smaller than $\Phi_{0}$ using a flux-lock system. A feedback circuit can measure a variation in the magnetic flux $\delta \Phi$ and it generates a flux $-\delta \Phi$ in order to keep the total flux of the SQUID constant. This 
feedback signal has the information of the flux variation in the superconductor loop and therefore it allows the MPMS to compute the magnetization of the sample.

Two types of measurements were carried out: magnetization versus temperature $(M-T)$ and magnetization versus magnetic field $(M-H)$, both of them with the magnetic field applied in the plane of the sample. Because of the small signal from the cobalt ultra thin film, separate measurements of the mounting straw and substrate were necessary in order to subtract the background. The general procedure for all the measurements was as follows:

The sample was centered on the pick up coils in order to obtain the maximum signal from it. We used two methods for centering: DC and RSO (Reciprocal Sample Option). For both methods an external magnetic field of 5 Tesla was applied to the sample in order to magnetize it. For the DC method, the sample was moved in one direction to locate roughly its center with respect of the coils. The RSO gives a more accurate measure of the center; where the sample is oscillated abound the center found with the DC scan at a fixed frequency, so that signal averaging is much more effective. This signal is processed with an algorithm in order to measure the magnetic response. The amplitude as well as the frequency of the oscillation in the RSO mode can be changed in order to maximize the signal and decrease the noise. Before the start of the experiment, the sample was demagnetized. This was carried out by alternating positive and negative magnetic fields to the sample at $350 \mathrm{~K}$ from $5000 \mathrm{Oe}$ and decreasing the field intensity to zero. Then the sample was zero-field cooled (ZFC) from $350 \mathrm{~K}$ to $2 \mathrm{~K}$. $M$-T measurements were carried out between $2 \mathrm{~K}$ and $350 \mathrm{~K}$ in a field of $1000 \mathrm{Oe}$. Finally, the sample was field-cooled (FC) from $350 \mathrm{~K}$ to $2 \mathrm{~K}$ in a field of $1000 \mathrm{Oe}$. $M$-T measurements were recorded during the process. The sample was then heated to $300 \mathrm{~K}$ and $M-H$ measurements were performed using a maximum magnetic field of $+/-7$ Tesla.

\section{Results and Discussion}

\subsection{Roughening of Cobalt Thin Films}


Several experiments were carried out to measure the roughness of cobalt films whose thickness ranged between $3.0 \mathrm{~nm}$ and $8.0 \mathrm{~nm}$, all of them deposited on of $\mathrm{Al}_{2} \mathrm{O}_{3}(11 \overline{2} 0)$.

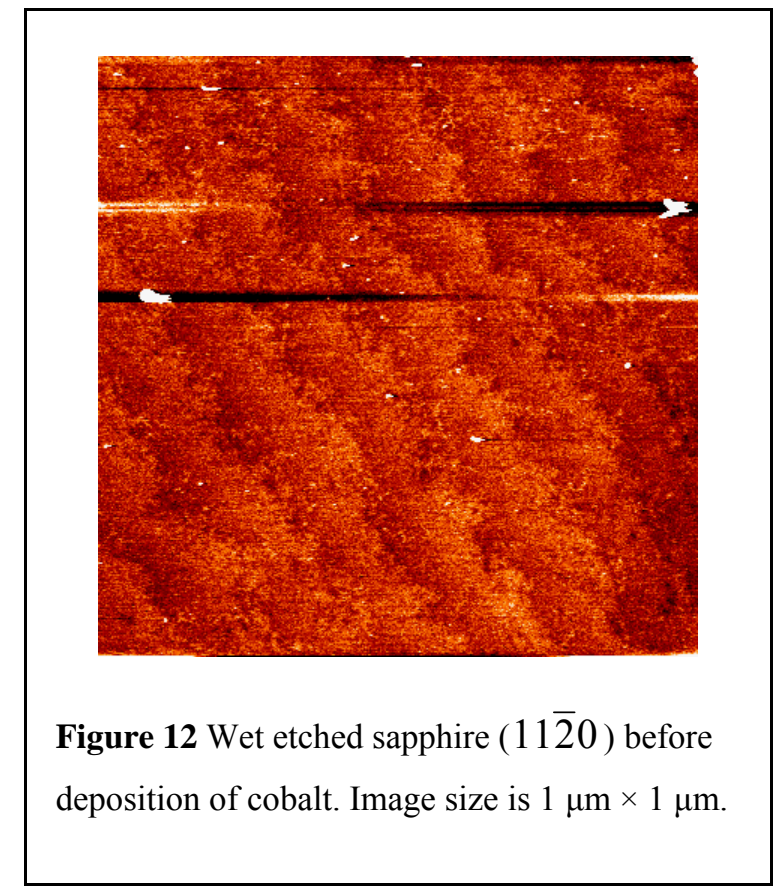

Here the results for one of the experiments with a cobalt film $5.0 \mathrm{~nm}$ thick are described. Figure 12 shows an AFM image of the substrate surface after the wet etching procedure. The surface depicts terraces with average widths of $\sim 120 \mathrm{~nm}$ and heights of $0.2 \mathrm{~nm}$. No multiple monoatomic steps are observed in the image because no annealing was performed to the substrate as observed previously. ${ }^{32}$ The out-of-plane roughness was measured to be $\sim 0.10 \mathrm{~nm}$; these parameters are a good indicator of a relatively clean surface. After this, $5.0 \mathrm{~nm}$ of cobalt were deposited on top of the sapphire at a temperature of $300{ }^{\circ} \mathrm{C}$. AFM images of the film after growth are shown in Figure 13. Each image was obtained by heating the sample in-situ on the AFM sample stage. For temperatures below $410{ }^{\circ} \mathrm{C}$, the film is very smooth with a roughness of $\sim 0.7 \mathrm{~nm}$. Above $410{ }^{\circ} \mathrm{C}$ there is a sudden formation of islands. At $\mathrm{T}_{\mathrm{a}}=504{ }^{\circ} \mathrm{C}$, we can see that the diameter of the islands increases as a result of coalescence, which produces an increase in the roughness of the film to $6.8 \mathrm{~nm}$. 
We did not observe the formation of rectangular pits as reported by Shi and Lederman $^{5}$ in this film nor in thinner or thicker ones $(3.0 \mathrm{~nm}<\mathrm{t}<8.0 \mathrm{~nm})$ where similar experiments were carried out. Therefore the mechanism for that pattern formation remains elusive.

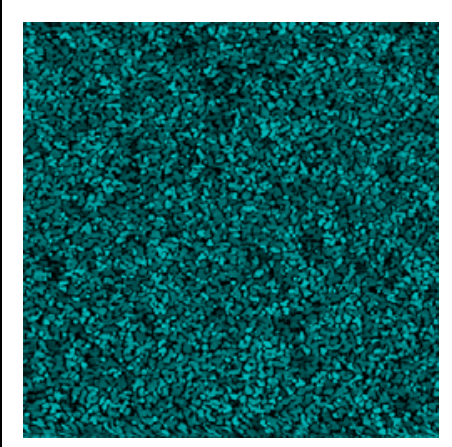

(a) $\mathrm{T}=34^{\circ} \mathrm{C}$

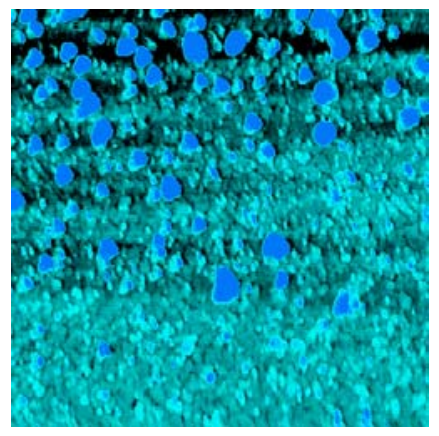

(d) $\mathrm{T}=462{ }^{\circ} \mathrm{C}$

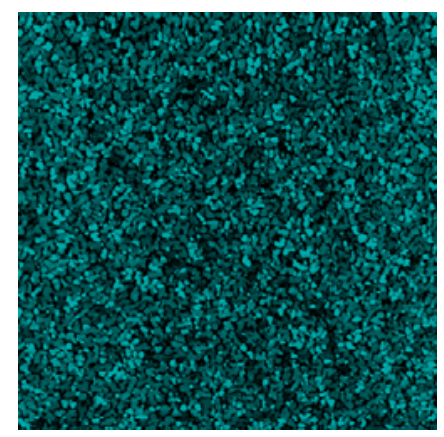

(b) $\mathrm{T}=201{ }^{\circ} \mathrm{C}$

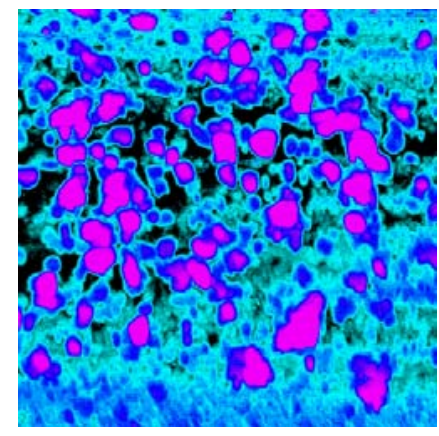

(e) $\mathrm{T}=482^{\circ} \mathrm{C}$

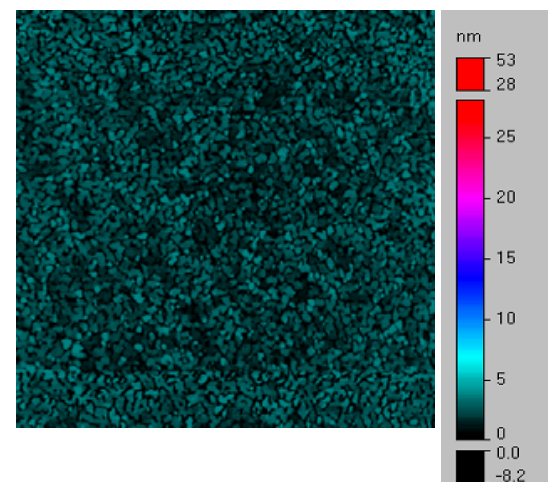

(c) $\mathrm{T}=410^{\circ} \mathrm{C}$

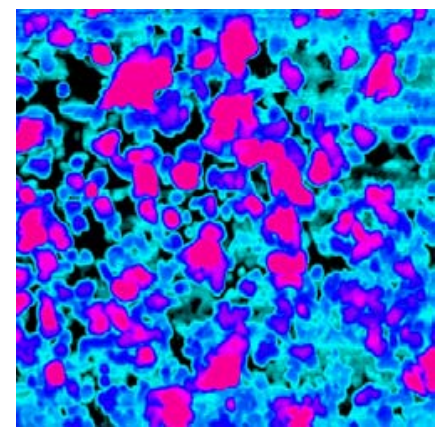

(f) $\mathrm{T}=504{ }^{\circ} \mathrm{C}$

Figure $13 \mathrm{In}$-situ AFM images of cobalt $(5.0 \mathrm{~nm})$ grown on $\mathrm{Al}_{2} \mathrm{O}_{3}(110)$ at different annealing temperatures. The color scale depicts the height of the islands in an axis perpendicular to the surface of the sample. The size of the images is $1 \mu \mathrm{m} \times 1 \mu \mathrm{m}$.

It is important to notice that the thermal equilibrium of the films was confirmed by a constant value of the resonance frequency for the needle sensor. In another experiment a $5.0 \mathrm{~nm}$ cobalt thin film was annealed at $600{ }^{\circ} \mathrm{C}$ and then cooled to room temperature to observe if there were any reversible processes in the roughening. In the graph of Figure 14 the roughness as a function of the annealing temperature $\left(T_{A}\right)$ for that 
experiment is shown. The roughening of the film starts at approximately $425{ }^{\circ} \mathrm{C}$ and continues to increase up to $5.5 \mathrm{~nm}$ at $600{ }^{\circ} \mathrm{C}$. After that point, the sample was cooled down. The roughness clearly continued increasing, despite decreasing the temperature. This gave us a clue that time might be an important variable in these types of experiments. This effect can be appreciated in Figure 15 where the roughness is plotted for three different $5.0 \mathrm{~nm}$ cobalt films, where different heating rates were used to reach $T_{A}$. For $T_{A}=475{ }^{\circ} \mathrm{C}$ (black squares) we observed a roughness of $1.21 \mathrm{~nm}$ for a rate of $0.38{ }^{\circ} \mathrm{C} / \mathrm{min}$, and a roughness of $4.74 \mathrm{~nm}$ for a rate of $0.17{ }^{\circ} \mathrm{C} / \mathrm{min}$. This means that for slower annealing rates the sample tends to be rougher than for faster annealing processes. The same behavior is observed at higher annealing temperatures of $500{ }^{\circ} \mathrm{C}$ and $525{ }^{\circ} \mathrm{C}$. We believe keeping a sample at a higher temperature causes the cobalt atoms to have a longer time to diffuse over the sapphire, and perhaps over the cobalt islands themselves, in order to form larger islands. For this reason, it is extremely difficult to make comparisons of absolute values of roughening in films with the same thickness, and furthermore to establish comparisons between films with different thicknesses. This is also problematic because time is a variable difficult to control when using large lateral scale AFM imaging, since in general the scan rates have to be slow for larger image sizes. Moreover imaging at high temperatures is difficult because occasionally the film morphology changes faster than the time it takes to scan a whole image. 


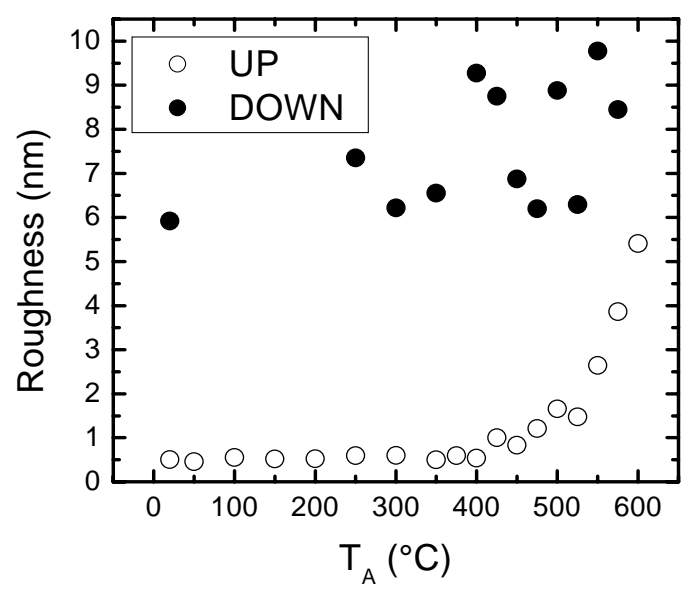

Figure 14 Roughening of a cobalt film $(5.0 \mathrm{~nm})$ on sapphire (110). The circles represent the roughness during heating and the dots during cooling.

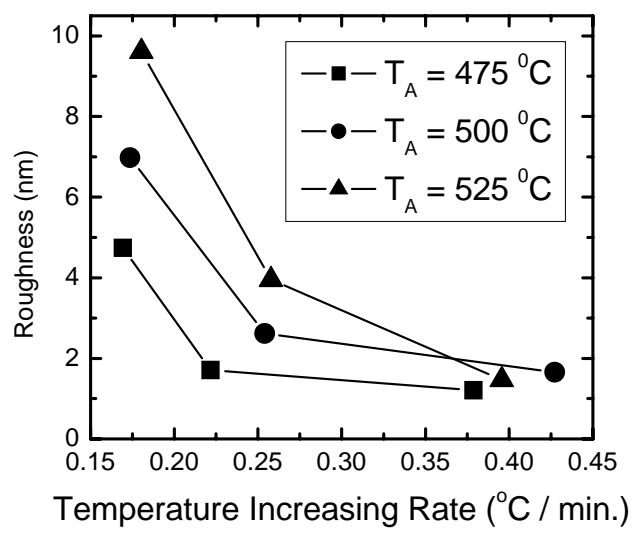

Figure 15 Roughness as a function of annealing rate for 3 different $5.0 \mathrm{~nm}$ thick Co film samples with different heating rates used to reach the annealing temperature $T_{A}$. 


\subsection{Cobalt ultra-thin film}

In-situ AFM images of the sapphire before and immediately after Co film growth, and
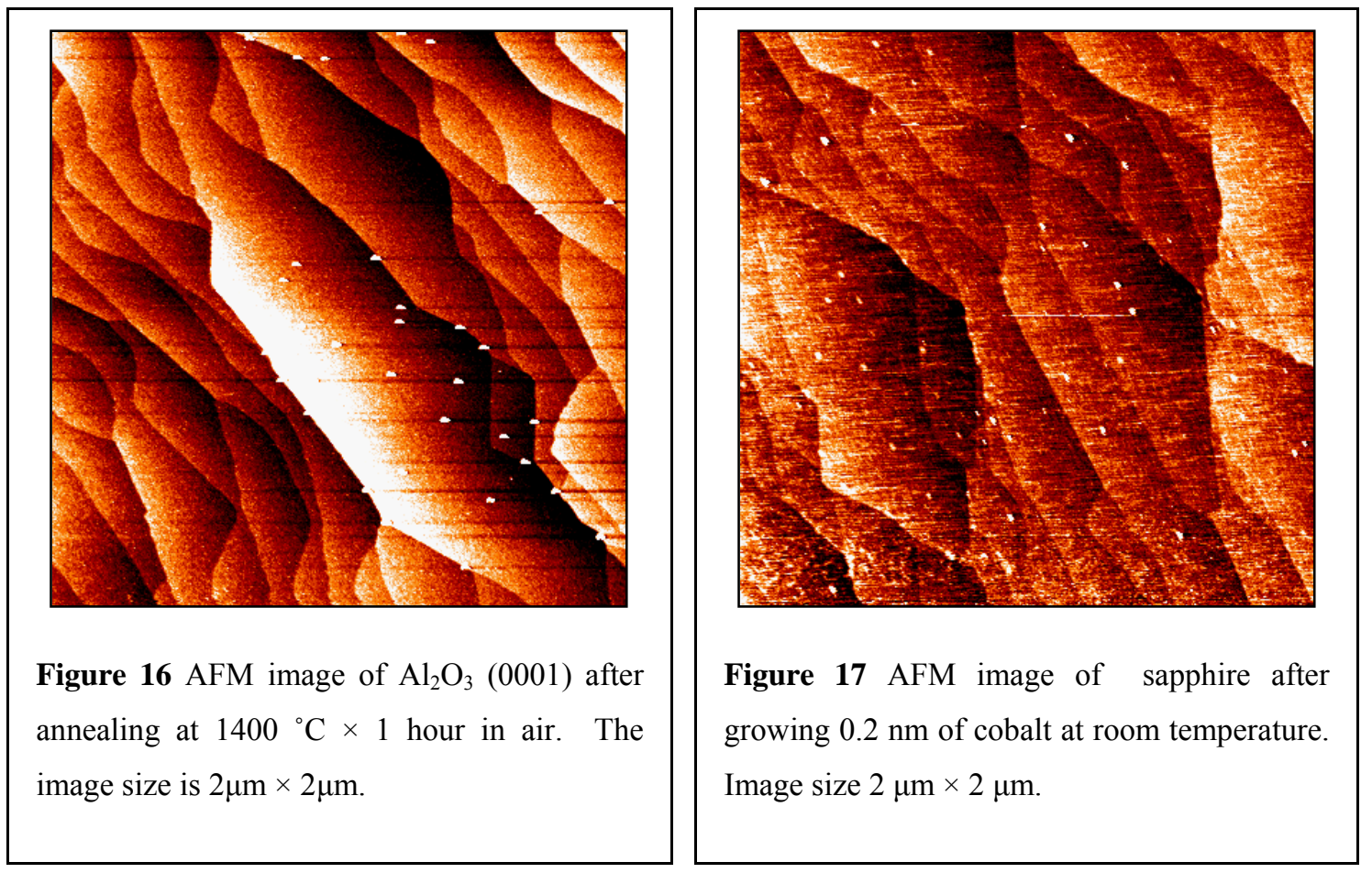

after annealing were obtained. All images were obtained in ultra high vacuum and they are not filtered unless otherwise noted. A clean $\mathrm{Al}_{2} \mathrm{O}_{3}(001)$ surface can be observed in Figure 16. Mono-atomic steps are well defined as a consequence of the annealing at $1400{ }^{\circ} \mathrm{C}$ in air. The RMS roughness along the axis perpendicular to the surface is $\sim 0.12$ $\mathrm{nm}$, and the atomic steps were measured to be approximately $0.2 \mathrm{~nm}$, in agreement with the interatomic distance of the substrate along the (001) direction. ${ }^{16}$ Figure 17 shows the AFM image of sapphire after growing $0.2 \mathrm{~nm}$ of $\mathrm{Co}$ at room temperature. The atomic steps of the substrate can still be observed in the image, which indicates that there is an ultra-thin film on top of the substrate. The surface roughness increased to $0.21 \mathrm{~nm}$. Figure 18 is an AFM image of the film after annealing at $400{ }^{\circ} \mathrm{C}$ on the AFM sample plate. It is possible to observe the formation of nanodots with an average diameter of $17.6 \pm 4.7 \mathrm{~nm}$ and an average height of $0.39 \pm 0.15 \mathrm{~nm}$. The histogram in Figure 19 reveals a wide distribution of diameters. It is not clear why the formation of nanodots was preferred to the formation of nanowires along the $\mathrm{Al}_{2} \mathrm{O}_{3}$ step-edges, as was previously 


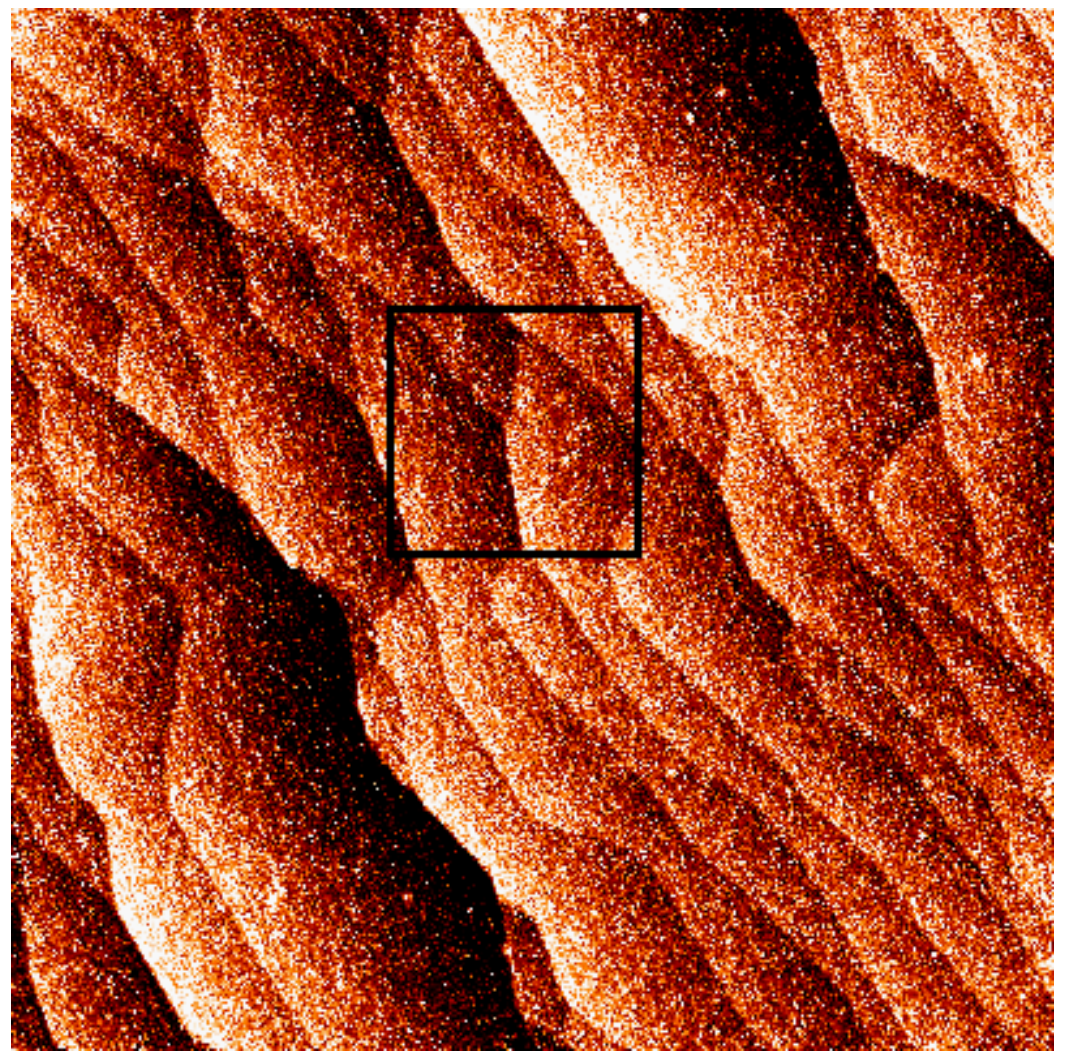

Figure 18 AFM image of $0.2 \mathrm{~nm}$ of Cobalt over sapphire (001) after annealing in ultra high vacuum at $400{ }^{\circ} \mathrm{C}$. The image shows the formation of nanodots with an average diameter of $17.7 \mathrm{~nm}$. The image size is $2 \mu \mathrm{m} \times 2 \mu \mathrm{m}$.

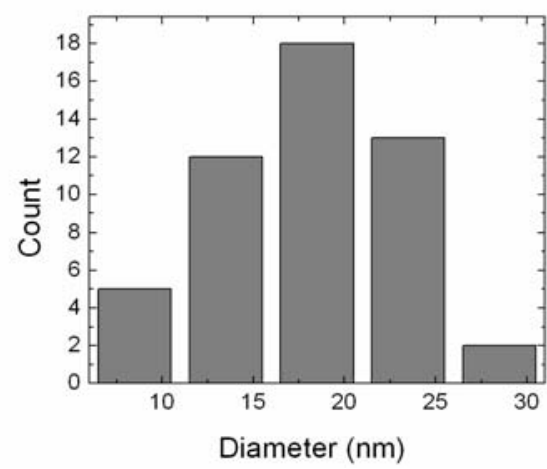

Figure 19 Histogram of the diameter of the nanodots obtained from the image in figure 20. Statistics are based on measurement of 50 nanodots.

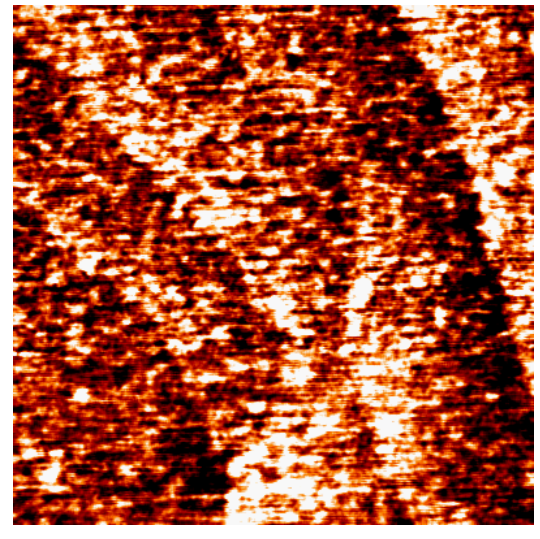

Figure 20 AFM image of cobalt nanodots. The image corresponds to the squared region of Figure 18. Image size: $500 \mathrm{~nm} \times$ $500 \mathrm{~nm}$. Image is slightly filtered. 
achieved in ${ }^{14}$ stepped W(110). Fe nanostripes were obtained in W(110) annealing the samples at $800 \mathrm{~K}$. In our case it is possible that the thermal energy of the cobalt atoms was not high enough to make them to diffuse towards the step edges of the sapphire. Future experiments using higher annealing temperatures are planned to test this hypothesis.

\subsubsection{Magnetic Properties of Ultra-Thin film}

The results for the magnetization of the ultra-thin film, after annealing, as a function of temperature with an external magnetic field applied parallel to the sample surface, are shown in Figure 21. The hysteresis in temperature between the zero-fieldcooled (ZFC) curve and the field-cooled (FC) measurements is evidence of

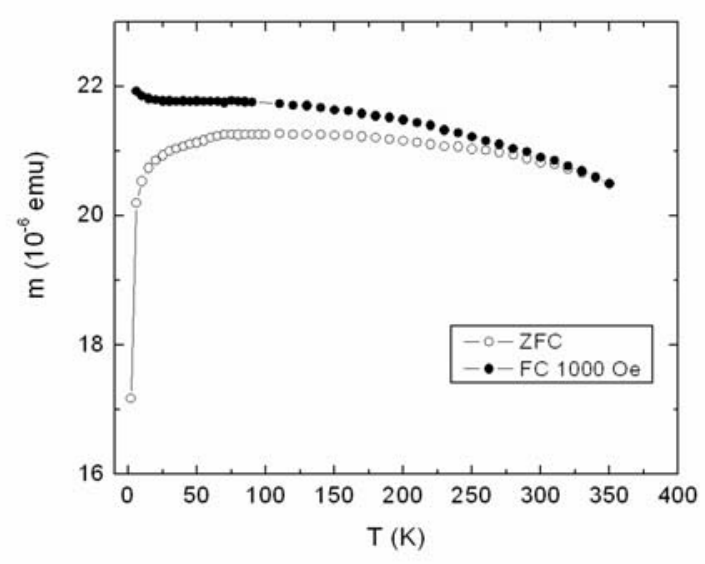

Figure 21 Magnetization versus temperature for the cobalt ultra-thin film. The black dots represent the magnetization for the field cooled sample and in white dots the magnetization for zero field cooled. The measurement field was 1000 Oe.

superparamagnetic behavior of the nanoparticles. In theory, the ZFC curve should peak at the blocking temperature of the nano-particles $\sim 130 \mathrm{~K}$. The broad ZFC curve is explained by the wide distribution in particle sizes. ${ }^{22}$ Previous experiments ${ }^{33}$ carried out 
on multilayers of $\mathrm{Co} / \mathrm{Al}_{2} \mathrm{O}_{3}$ with $t_{\mathrm{Co}}=0.1,0.3$ and $0.7 \mathrm{~nm}$, obtained with sequential sputtering of $\mathrm{Co}$ and $\mathrm{Al}_{2} \mathrm{O}_{3}$, also have shown superparamagnetic behavior of the films with a blocking temperature of $\sim 7 \mathrm{~K}$ for an average diameter of particles equal to $1.4 \mathrm{~nm}$. Our larger $T_{B}$ can be explained with the relaxation time ${ }^{21}$

$$
\tau=\tau_{0} \exp \left(\frac{K_{e f f} V}{k_{B} T}\right)
$$

where $\tau$ is the average time for switching the magnetization of a nanoparticle with an anisotropy energy density $K_{\text {eff }}$ and a volume $V$. The thermal switching of the magnetization occurs at the blocking temperature when $\tau\left(\sim 10^{-10}\right.$ to $10^{-13} \mathrm{sec}$. for temperatures above $T_{B}$ ) is equal to the experimental time $\sim 150 \mathrm{sec}$. Notice that the magnitude of $T_{B}$ depends on the volume of the nanoparticles. For larger nanoparticle volume a larger blocking temperature is expected. In our case $T_{B}=130 \mathrm{~K}$ for an average nanoparticle diameter of $17.6 \mathrm{~nm}$.

Figure 22 shows the magnetization versus magnetic field of the ultra-thin film above the blocking temperature ( $300 \mathrm{~K}$ ) with $H$ applied parallel to the sample surface. We fitted the Langevin function to the data, as explained in Chapter 1, using $\mu_{p}$ as a fitting parameter. The result was $(1.60 \pm 0.07) \times 10^{4} \mu_{B}$ for each nanodot. Given this value the number of Bohr magnetons for each cobalt atom can be estimated. Considering each nanoparticle as a cylinder with a diameter of $17.6 \mathrm{~nm}$ and an average height of $0.39 \mathrm{~nm}$ (determined from the AFM images), an average volume of $95.1 \pm 63.5 \mathrm{~nm}^{3}$ is obtained. For the fcc cobalt with lattice parameter of $0.3528 \mathrm{~nm}^{34}$ (each containing 4 atoms) we should have a volume of $4.39 \times 10^{3} \mathrm{~nm}^{3}$ and therefore $\sim 2.16 \times 10^{3}$ fcc unit cells in each nanoparticle, each nanoparticle containing $\sim 8.65 \times 10^{3}$ atoms. Dividing the number of Bohr magnetons per nanoparticle by the number of atoms per nanoparticle, an average magnetic moment of $1.85 \pm 1.23 \mu_{B}$ per cobalt atom is achieved. In the literature the magnetic moment for the bulk cobalt is ${ }^{34} 1.72 \mu_{B}$, which represents a difference of $8.8 \%$ with respect to our ultrathin film. 


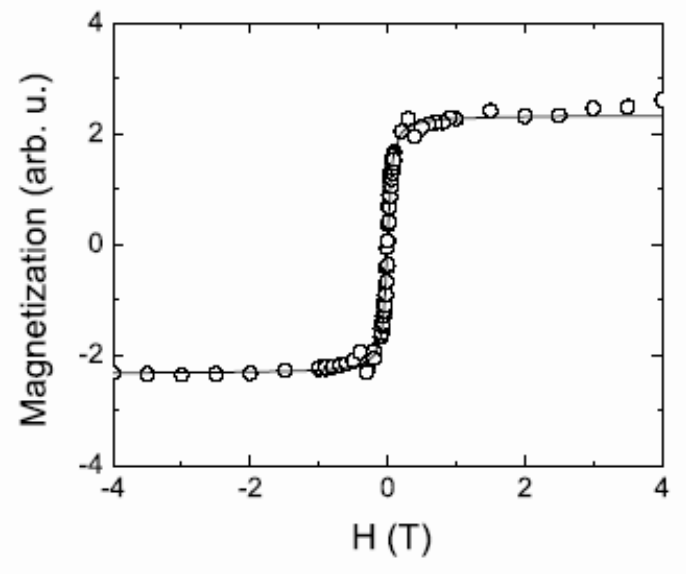

Figure 22 Magnetization as a function of magnetic field for the ultra-thin film taken at $300 \mathrm{~K}$. The line corresponds to the fitting of the Langevin function to the experimental data.

The larger magnetic moment of the cobalt atoms in the nanodots might be due to an underestimation in our calculation of the average volume for the nano-particles or to a real enhancement in the magnetic moment per atom. This enlargement has been recently measured in submonolayer cobalt films grown on $\operatorname{Pt}(111)$ and measured using X-ray magnetic circular dichroism ${ }^{35}$ and in colloidal Co nanoparticles. ${ }^{36}$ The effect is explained by an increase in the orbital magnetic moment of cobalt atoms on the surface, leading to a larger total magnetic moment per atom. A transition metal atom located on a surface has $d$-electrons that are more localized than in the bulk material because there are fewer nearest neighbors, and therefore its atomic-like behavior is enhanced. This would decrease the quenching of orbital angular momentum effect observed in the bulk and also increase the density of states of the majority spins at the Fermi level. ${ }^{37}$ In our case we might expect that atoms located in the surface of the nano-particles would contribute with larger orbital magnetic moment, and also increase the net magnetization due to the higher density of states. Experiments with smaller nanoparticle sizes and with samples with smaller size distributions are necessary to settle this issue. In addition, measurements 
using magnetic x-ray circular dichroism, where the magnetic moment can be measured, would also be useful.

\section{Conclusions}

In the present work the roughening during annealing of $5.0 \mathrm{~nm}$ Co films grown on $\mathrm{Al}_{2} \mathrm{O}_{3}$ (110) was studied. The films showed a roughening transition at a temperature of $410{ }^{\circ} \mathrm{C}$, at which the surface roughness increased rapidly with increasing annealing temperature. The roughening is not reversible because the samples kept their roughening state after cooling them down. Moreover the roughening process has a strong time dependence, which was observed using different annealing rates for the films. In another set of experiments, a submonolayer cobalt film was deposited on the surface of stepped $\mathrm{Al}_{2} \mathrm{O}_{3}$ (001). The sample showed the formation of nanodots with an average diameter of $17 \mathrm{~nm}$ after annealing at $400{ }^{\circ} \mathrm{C}$. Magnetization as a function of temperature measurement showed that the sample was superparamagnetic. The ZFC curve peaked at a temperature of $130{ }^{\circ} \mathrm{C}$, which would correspond to the average blocking temperature of the particles. The ZFC curve is very wide as a result a wide distribution in particles sizes, as confirmed by AFM images. By fitting the Langevin function for MH curves at $300 \mathrm{~K}$ an average magnetic moment per nano-particle of $1.60 \times 10^{4} \mu_{B}$ was calculated, which is equivalent to a magnetic moment per cobalt atom of $1.85 \mu_{B}$. This value is $8.8 \%$ larger than for bulk HCP cobalt. This enhancement may be the result of an increase of the orbital magnetic moment of the atoms located at the surface of the nanoparticles and an increase the density of states of the majority spins at the Fermi level as observed by other groups. To determine whether this enhancement is real, it is necessary to decrease the size and distribution of the particles, which may be achieved by annealing at higher temperatures, and to perform x-ray dichroism absorption measurements. 


\section{References}

${ }^{1}$ C. P. Bean, J. Appl. Phys. 26, 1381 (1955).

${ }^{2}$ B. Nonas, I. Cabria, R. Zeller, and P. H. Dederichs, Phys. Rev. Lett. 86, 2146 (2001).

${ }^{3}$ Constitution of Binary Alloys, edited by M. Hansen (McGraw-Hill, New York, 1958), p. $471 \mathrm{ff}$.

${ }^{4}$ Ch. Morawe, A. Stierle, N. Metoki, K. Bröhl and H. Zabel, J. Magn. Magn. Mater. 102, 223 (1991).

${ }^{5}$ H. Shi and D. Lederman, J. Appl. Phys. 87, 6095 (2000).

${ }^{6}$ H. T. Shi and D. Lederman, Phys. Rev. B 58, R1778 (1998).

${ }^{7}$ R. F. Pease, in Proceedings of the 1991 Symposium on Nanostructures and Mesoscopic Systems, W. P. Kirk and M. A. Reed, eds. (Academic Press 1992).

${ }^{8}$ A. Westphalen, H. Zabel and K. Theis-Bröhl, Thin Solid Films 449, 207 (2004).

${ }^{9}$ D. J. Sellmyer, M. Zheng and R. Skomski, J. Phys.: Condens. Matter 13, R433 (2001).

${ }^{10}$ M. Kröll, W. J. Balu, D. Grandjean, R. E. Benfield, F. Luis, P. M. Paulus, and L. J. de Jongh, J. Magn. Magn. Mater. 249, 241 (2002).

${ }^{11}$ H. Zeng, R. Skomski, L. Menon, Y. Liu, S. Bandyopadhyay, and D. Sellmeyer, Phys. Rev. B 65, 134426 (2002).

${ }^{12}$ A. Kazadi Mukenga Bantu and J. Rivas, J. Appl. Phys. 89, 3393 (2001).

${ }^{13}$ R. Moroni, D. Sekiba, F. Buatier de Mongeot, G. Gonella, C. Boragno, L. Mattera, and U. Valbusa, Phys. Rev. Lett. 91, 1672071 (2003).

${ }^{14}$ J. Hauschild, H. J. Elmers, and U. Gradmann, Phys. Rev. B 57, R677 (1998).

${ }^{15}$ M. Yoshimoto, T. Maeda, T. Ohnishi, H. Koinuma, O. Ishiyama, M. Shinohara, M. Kubo, R. Miura and A. Miyamoto, Appl. Phys. Lett. 67, 2615 (1995).

${ }^{16}$ L. Pham Van, O. Kurnosikov and J. Cousty, Surf. Sci. 411, 263 (1998).

${ }^{17}$ O. Kurnosikov, L. Pham Van and J. Cousty, Surf. Sci. 459, 256 (2000).

${ }^{18}$ Y. Shiratsuchi, M. Yamamoto and Y. Kamada, Jpn. J. Appl. Phys. 41, 5719 (2002).

${ }^{19}$ F. J. Himpsel, T. Jung, A. Kirakosian, J. L. Lin, D. Y. Petrovykh, H. Rauscher and J. Viernow, MRS Bulletin 24, 20 (1999).

${ }^{20}$ Y. Shiratsuchi, M. Yamamoto, Y. Endo, D. Li and S. D. Bader, J. Appl. Phys. 94, $7675,(2003)$. 
${ }^{21}$ A. H. Morrish, The Physical Principles of Magnetism, Reprint of the $1^{\text {st }}$ ed. (Wiley, New York, 1980).

${ }^{22}$ A. Punnoose, T. Phanthavady, M. S. Seehra, N. Shah and G. P. Huffman, Phys. Rev. B 69, 54425, (2004).

${ }^{23}$ F. Dwikusuma, D. Saulys and T. F. Kuech, J. Electrochem. Soc., 149, G603 (2002).

${ }^{24}$ F. J. Giessibl, Science 267, 68 (1995).

${ }^{25}$ J. Wang, A. Howard, R. G. Egdell, J. B. Pethica and J. S. Foord, Surf. Sci., 505, 337 (2002).

${ }^{26}$ A. Michels, F. Meinen, T. Murdfield, W. Gohde, U. C. Fisher, E. Beckmann and H. Fuchs, Thin Solid Films, 264, 172 (1995).

${ }^{27}$ W. Clauss, J. Zhang, D. J. Bergeron and A.T. Johnson, J. Vac. Sci. Technol., B17, 1309 (1999).

${ }^{28}$ B. D. Josephson, Phys. Letters 1, 251 (1962).

${ }^{29}$ P. W. Anderson and J. M. Rowell, Phys. Rev. Lett. 11, 80 (1963).

${ }^{30}$ C. Kittel, Introduction to Solid State Physics, $6^{\text {th }}$ ed. (John Wiley and Sons, Inc., N.Y.) 1986.

${ }^{31}$ Quantum Design, MPMS Hardware User's Manual (1999).

${ }^{32}$ B. Wolfing, K. Theis-Brohl, C. Sutter and H. Zabel, J. Phys.: Condens. Matter, 11, 2669 (1999).

${ }^{33}$ J. M. Torres, F. Luis, L. M. Garcia, J. Bartolome, J. Stankiewicz, F. Petroff, F. Fettar and A. Vaures, J. Magn. Magn. Matter. 242-245, 575 (2002).

${ }^{34}$ Landolt-Bornstein, Numerical Data and Functional Relationships, Sci. and Tech. 19 (Springer-Verlag, Berlin), 1986.

${ }^{35}$ A. Hahlin, J. Hunter Dunn, O. Karis, P. Poulopoulos, R. Nunthel, J. Lindner, and D. Arvanitis, J. Phys. Condens. Matter, 15, S573 (2003).

${ }^{36}$ J. P. Chen, C. M. Sorensen, K. J. Klabunde and G. C. Hadjipanayis, Phys. Rev. B 51, 11527 (1995).

${ }^{37}$ P. Gambardella, S. Rusponi, M. Veronese, S. S. Dhesi, C. Grazioli, A. Dallmeyer, I. Cabria, R. Zeller, P. H. Dederichs, D. Kern, C. Carbone and H. Brune, Science 300, 1130 (2003). 